\title{
CLINICAL EVALUATION OF \\ PERIODONTAL REGENERATIVE TECHNIQUE AND ORTHODONTIC THERAPY IN MANAGEMENT OF PATHOLOGICALLY MIGRATED TOOTH
}

\author{
ABEER S. GAWISH* AHMED E. SALAMA* \\ HISHAM.S SADEK ${ }^{* *}$
}

\section{ABSTRACT}

Pathologic migration is defined as a change in tooth position resulting from disruption of forces which maintain tooth in a normal position.

Pathologic migration of tooth due to periodontal disease is considered as one aspect of esthetic problems.

The purpose of the present study's, to evaluate a combined periodontal regenerative procedure with orthodontic therapy in order to solve functional and esthetic problems in migrated periodontally affected teeth.

Fourteen patients suffering from extruded maxillary incisors with 15 intra-bony defects have probing depth (PD)7.7mm due to advanced periodontal disease were included in the present study. At base line $P D$, clinical attachment level (CAL) and radiographic examination were performed. All patients received periodontal therapy, which include elevation of periodontal flap (papilla preservation technique) with Demineralized freeze dried bone allograft (DFDB).

\footnotetext{
* lecturer, Oral Medicine, Periodontology ,Oral Diagnosis and Radiology department, Faculty of Dental Medicine (girls section) Al Azhar, University.

${ }^{* *}$ Lecturer, Orthodontic department, Faculty of Dental Medicine(girls section) Al Azhar, University

${ }^{* * *}$ Lecturer, Oral Medicine, Periodontology department, Faculty of Oral \& Dental Medicine , Cairo , University
} 
Active orthodontic treatment was started after one week from the surgery using the segmented arch technique, in order to intrude the moved teeth into the defects. Maintenance therapy was performed every month unti orthodontic treatment was completed.

At the end of the study period (one year) all the clinical parameters were reevaluated

At the end of treatment, the value of mean $P D$ reduction was $4.1+1.39 \mathrm{~mm}$. Mean gain of CAL was $5.32+1.6 \mathrm{~mm}$. All differences were highly significant. Radiological examination revealed a favorable bone fill of all defects. Moreover, after the completion of orthodontic treatment a satisfactory realignment of the pathologically extruded teeth were achieved.

The present study for the combined regenerative periodontal technique and orthodontic therapy performed to treat extruded teeth

with intra-bony defects, showed a highly significant probing depth reduction, gain in clinical attachment and favorable radiological bone fill.

\section{INTRODUCTION}

Chronic periodontitis, one of the most common diseases of human, is an infectious condition that can result in the inflammatory destruction of periodontal ligament and alveolar bone (1)

Destruction of tooth supporting connective tissue is a crucial stage in the progression of periodontal disease (2).

Pathologic migration of tooth due to destruction of tooth supporting structures is a common chief complaint of chronic periodontitis patients and often motivates to seek dental treatment ( $3 \& 4)$

The main goal of periodontal therapy is to restore periodontal tissues, essentially bone, cementum and periodontal ligament lost during periodontal disease (5).

The periodontal regenerative procedure including a variety of surgical techniques with bone grafts; bone substitutes and guided tissue regeneration GTR or a combination between them (6). periodontal surgery as part of the treatment of periodontal disease is performed to gain access to diseased area for adequate cleaning; to achieve pocket reduction; and to restore the periodontal tissues lost through the disease (7) 
Moreover orthodontic therapy may contribute to improved periodontal status of pathologically migrated tooth when orthodontically induced forces and oral hygiene are well controlled (8) . Long term stability has been reported of periodontally compromised patients following combined orthodontic-periodontic treatment (9).

One of the most important factors to obtain a good treatment outcome in patients suffered from reduced periodontal support, is the use of light and continuous orthodontic forces, in absence of gingival inflammation .Furthermore the combination between periodontal treatment and orthodontic intrusion may result in new attachment formation and clinical attachment gain if good oral hygiene is maintained (10\&11).Polson etal., 1984(12) reported in an experimental study on infrabony defect, that no detrimental effect on the attachment level is observed if complete erradication of subgingival infection is performed before orthodontic movements is begun.

Periodontal therapy of pathologically migrated teeth can , in some instances, induce a reactive repositioning movement of teeth within the dental arch and this may be due to the elimination of pathologic granulation tissues during treatment (13)

The clinical investigations that have studied the effect of orthodontic movement in patients with intrabony defects are limited and results obtained are different $(14,15,16,17,18)$

The present study was conducted to evaluate the clinical outcome of a combined approach, including periodontal surgery with bone graft and orthodontic intrusion, in the treatment of patients suffering from intrabony osseous defects on extruded maxillary anterior incisors.

\section{Subjects and Methods}

Fourteen patients presenting 15 diastema secondary to pathologic migration due to periodontal involvement and intrabony defects were participated in this study. Ten patients were female and four were male there age ranged from 38 to 55 years old.

Subjects were selected from patients with severe periodontal disease seeking treatment in periodontal clinic in Alzhraa hospital, faculty of dental Medicine (Girls) Al-Azhar university. Patients were systematically examined for the presence of recently formed diastema associated with a loss of periodontal support. The clinical inclusion criteria were : 1) migration with at least $1 \mathrm{~mm}$ of extrusion of one maxillary incisor in respect to the incisal edge of the contralateral incisor; 2) radiological evidence of intrabony defect and the mesial aspect of the extruded incisor; 3) probing depth (PD) $\geq 6 \mathrm{~mm}$ at the defect site and 4) plaque index $(\mathrm{PI}) \leq 15 \%$ (19) . 
Eleven central and four lateral incisors were included in the study. The patients were asked to provide a picture of their dentition before the initiation of the pathologic drift. At base line all clinical parameters were performed include, PD measured at six points on each tooth from the free gingival margin to the point of the deepest probe penetration and clinical attachment (CAL)which is the distance between the cemento-enamel junction (CEJ) and the bottom of the pocket were assessed using a standardized graduated periodontal (20). Intra-oral periapical radiographs were obtained following the long cone parallel technique fig (1). Clinical photographs were also have been taken fig (2)

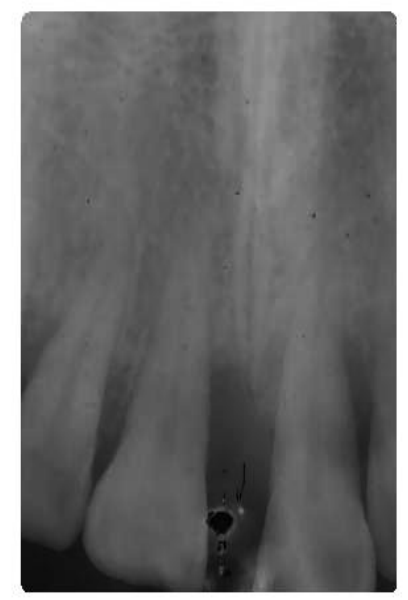

Fig.1 Radiographic documentation showed the bone defect at base line examination

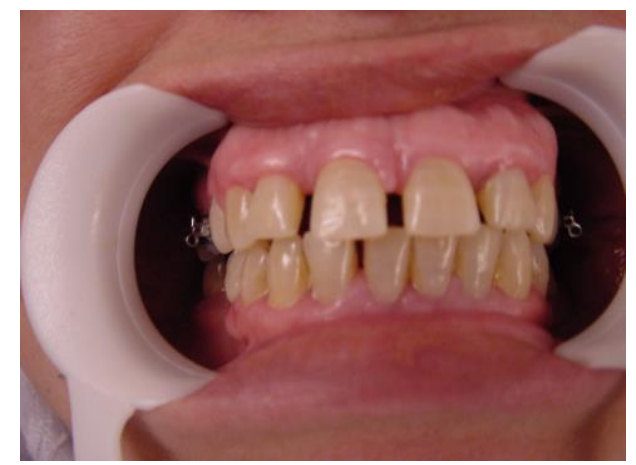

Fig 2. Clinical photograph before treatment 


\section{Initial periodontal therapy}

All patients received initial treatment consisted of scaling and root planing, occlusal adjustment and oral hygiene instructions were performed in 2 or 4 appointment with sharpened hand instruments. The patients were re-evaluated 6 weeks after the completion of the last scaling and root planning session.

\section{Surgical Procedures}

All patients received surgical therapy with bone graft. The flap design was "papilla preservation technique"(21) to obtain good esthetic results fig (3).

A buccal and interproximal intrasulcular incision to the alveolar bone crest, involving the two teeth neighbouring the defect with maximum caution to preserve the interdental papilla. A full thickness flap was elevated to the level of the buccal alveolar crest. The buccal and interproximal incision was continued intrasulcularly to reach and extended to the palatal aspect. The defect was fully debrided from the granulation tissue and scaling and root planing was performed fig (4).

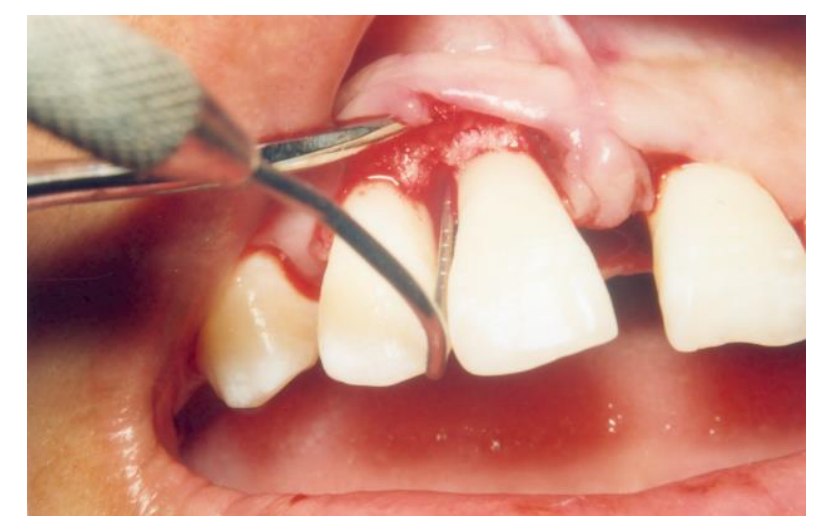

Fig 3. Papilla preservation flap was elevated 


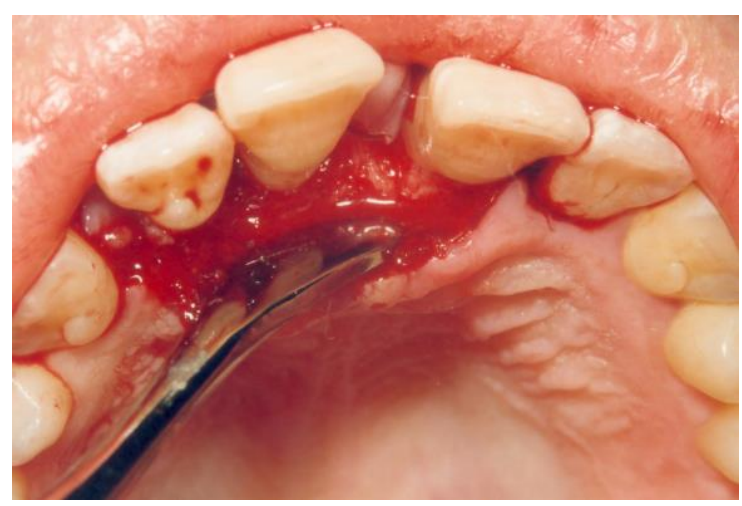

Fig 4. Scaling and root planing of the intrabony defect was performed

The defects were filled with Demineralized freez dried bone allograft*; care was taken not to overfill the defects fig (5).

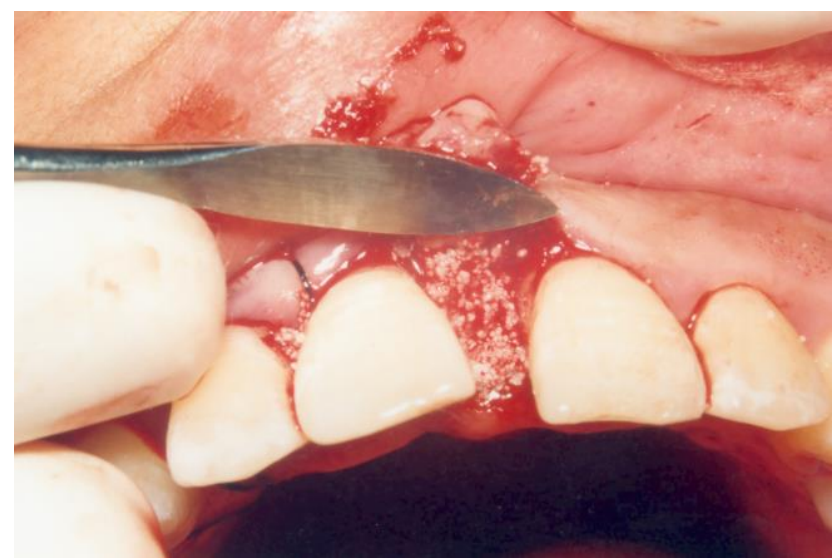

Fig 5. The defect filled with Demineralized freez dried bone allograft ( DFDBA)

The flaps were sutured at the original levels using a 3-0 silk. Post surgical medications included $500 \mathrm{mg}$ amoxacillin, twice daily for 6 days, $200 \mathrm{mg}$ brufen analgesics and $0.2 \%$ cholorohexidine mouth rinses two times daily for 2 weeks. One week later, the sutures were removed and patients started mechanical cleaning. At that time, the active orthodontic treatment started following the segmented arch technique (22\&23). 
A biomechanical system (st .wire, Roth system 0.22$)^{* *}$ made of intrusive arches and continuous forces in order to close spaces and to obtain intrusion of the pathologically extruded teeth.

The posterior anchorage was obtained by means of palatal arches and stainless steel segments.

During the orthodontic treatment period, all patients were enrolled in a recall program with maintenance therapy including professional tooth cleaning every 2 to 3 months (24). Orthodontic treatment lasted for one year. At the end of the treatment period, all patients had received permanent retention using Maryland fixed splint in order to avoid orthodontic relapse and for better chewing comfort.

At the same time clinical and radiographic parameters were measured.

The extent of intrusion was assessed in relation to the contralateral incisor edge by measurements on study models casts using a calibrated calliper .

The patients were seen every week for 4 weeks and then once a month for 1 year after .During these maintenance visits, oral hygiene instructions were reinforced and supragingival scaling was performed. All clinical parameters which includes PD,CAL, radiographs and clinical photographs were taken at base line fig (1\&2) and one year after fig (8\&9).At the end of the study period both pre and post operative mean values and standard deviations were calculated. Differences were statistically

analysed by mean of $\mathrm{t}$ - test. Values with $\mathrm{P}<0.001$ were considered statistically significant

\section{RESULTS}

Prior to treatment the PD at the diastema sites were ranged from 6 to $9.5 \mathrm{~mm}$ and their mean was $7.17 \pm 1.36 \mathrm{~mm}$, after treatment, it reaches $3.07 \pm 1.59 \mathrm{~mm}$

The mean reduction of the PD was $4.1 \pm 1.39 \mathrm{~mm}$. The statistical analysis of the data at base line and the end of the study period revealed a high significant difference in the reduction of PD table (1) fig (6)

\footnotetext{
${ }^{* *}$ Cera-med,AlloGro.Germany

**OPMI - ORL, Carl zeiss, obel ochen, Germany
} 
Table. 1 Mean reduction of PD values at base line and One year

\begin{tabular}{|c|c|c|c|} 
Pocket depth & BASE line & One year & reduction \\
\hline MEAN & 7.17 & 3.07 & 4.10 \\
\hline SD & 1.36 & 0.59 & 1.39 \\
\hline & & & \\
\hline P VALUE & 0.001 & & \\
\hline
\end{tabular}

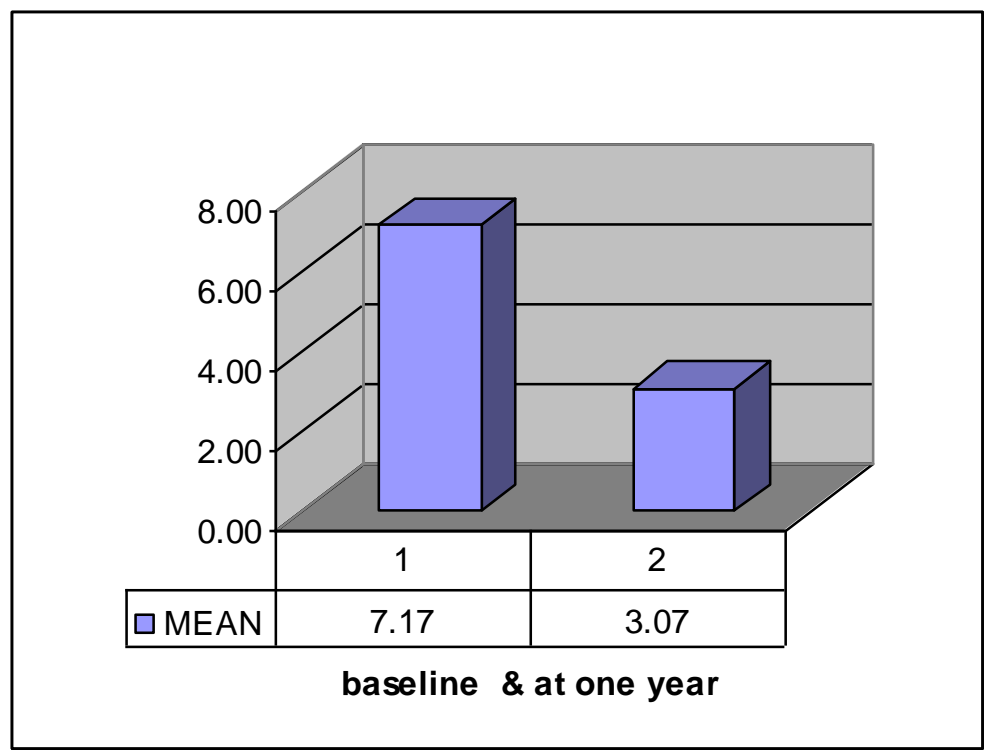

Fig. 6 The mean PD at baseline and at one year period

Table (2) fig (7) showed that CAL ranged from 7 to $12.5 \mathrm{~mm}$ before treatment and from 3 to $6 \mathrm{~mm}$ after treatment. The mean CAL at base line was $9.61 \pm 1.61$ and it reaches $4.29 \pm 0.99$ with mean gain in CAL of about $5.32 \pm 1.6$ at the end of the study period. This difference was statistically highly significant.

The radiographic examination showed a favorable bone fill of all vertical defects fig (8). 
The difference between pre and post treatment values of all the measured parameters was statistically highly significant $(\mathrm{P}<0.001)$. No negative effects due to orthodontic movement were observed on the treated teeth (i.e., root resorption, loss of pulp vitality, and compensatory extrusion of the neighboring teeth).

Table. 2 Mean values in $\mathrm{mm}$ and the gain of CAL at the base line and one year

\begin{tabular}{|c|c|c|c|} 
CAL & BASE line & One year & Gain of CAL \\
\hline MEAN & 9.61 & 4.29 & 5.32 \\
\hline SD & 1.61 & 0.99 & 1.60 \\
\hline & & & \\
\hline P VALUE & 0.001 & & \\
\hline
\end{tabular}

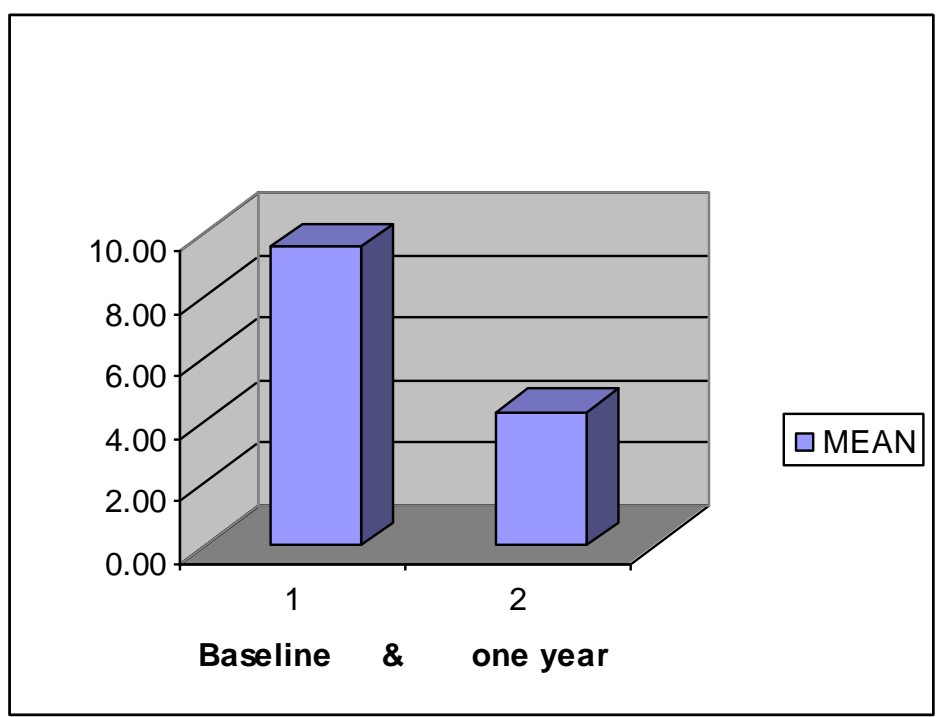

Fig 7. The mean value of CAL at baseline and at one year 
Egyptian

Orthodontic Journal

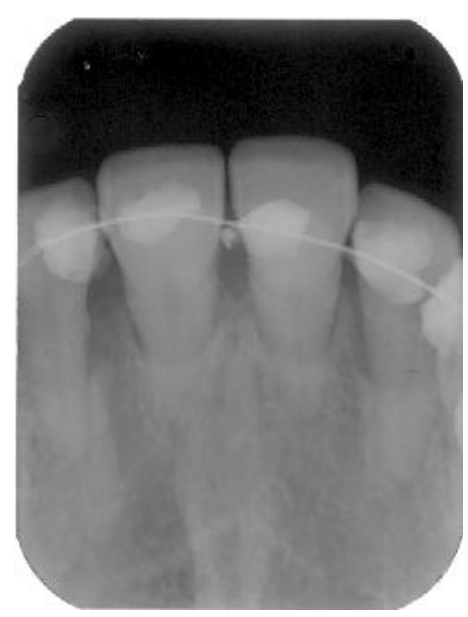

Fig. 8 Radiographic documentation showed a favorable bone fill.

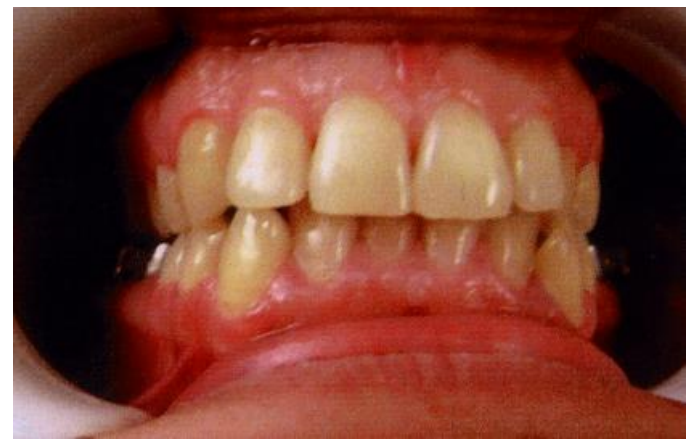

Fig. 9 Clinical photograph following orthodontic treatment

\section{DISCUSSION}

The present study was carried out to evaluate the possibility of performing orthodontic movement to migrated anterior teeth with intrabony defects in subjects suffering from advanced periodontal disease. The golden standard in the periodontal therapy is to stop disease progression and to regenerate the lost periodontal support over the years. Open flap surgery, graft materials and regenerative techniques have been used to achieve this goal in periodontal lesions with intrabony pockets $(25 \& 26)$ 
Periodontal surgery was performed first; in order to facilitate the removal of the granulation tissue in the intrabony defect. The technique used in this study was papilla preservation surgery with bone graft and orthodontic intrusion., resulted in the realignment of the treated incisors with radiological bone fill of the defect, CAL gain and PD reduction. The application of papilla preservation technique was a regenerative procedures allowed complete coverage of the defect and obtain good protection of the regenerating tissue through their complete coverage of the graft with the flap. Moreover it offers a better esthetic results specially in the anterior teeth (21). The bone graft used to augment the regeneration of bone in the present study was DFDBA which has an osteoinductive properties induce new bone formation and periodontal regeneration and enhance new attachment apparatus formation (27\&28). The absence of any clinical inflammation such as bleeding on probing confirmed the possibility of achieving a clinically healthy periodontal condition. Cardarpoli etal 2001(29) obtained positive results in terms of shortening the clinical crown length, affecting intrusion and increasing the marginal bone level, without significant apex resorption .Chronic periodontitis may result in periodontal lesion with intraosseous defects and extrusion of the upper incisors, orthodontic treatment may be a reliable therapeutic approach to realign migrated teeth once periodontal therapy is performed.

There are only few studies and case reports regarding the use of orthodontic movement in treatment of pathologically migrated teeth with infrabony pockets $(14,15,16,17,18 \& 30)$

Experimental studies on animals confirmed that orthodontic treatment has no detrimental effect on periodontal tissues if plaque control is provided but if there is any inflammation a further periodontal breakdown will result. Different results have been reported regarding the periodontal healing. These results may vary from loss of clinical attachment level around orthodontically moved teeth, which were periodontally involved (30) or no connective tissue attachment loss (12) .

However, in both studies no intrusive mechanic was used, but the teeth were tilted or bodily moved into the defects. On the other hand

Melsen etal .,1989 (11)in his exprimental study demonstrated a new attachment gain through surgical periodontal treatment and orthodontic intrusion in a situation of induced periodontal disease without infrabony pockets. Furthermore Geraci etal 1990 (31) demonstrated reattachment of the periodontium after bodily movement of the teeth into infrabony lesions. Ingber 
1974(32) studied the use of extrusive movement in order to realign the crestal bone level in the treatment of migrated periodontally affected teeth. However, endodontic and restorative treatment to reestablish a normal outline of the incisal margin was necessary at the end of this study.

From the above mentioned studies it seems clear that the use of intrusive movement and biologically conservative means to realign extruded teeth following periodontal disease were more effective (11\&30).

Intrusive orthodontic movement was used in the present study to realign extruded upper incisors in patients with chronic periodontitis characterized by presence of infrabony defects.

The results of the present study showed a highly significant decrease in PD and CAL gain, with fill of bone defects. Clinical attachment gain exceeded probing depth reduction, with a decrease in gingival recession probably associated with the intrusive movement.

These results were superior than the results obtained from Corrente etal 2003 (33)who used a combined surgical erradication of the infrabony defect without use of bone grafts. In the present study bone graft enhance the regeneration of lost periodontal support of the intrabony defect present.

Effective coronal shift of the soft tissues, seems to be obtained by early start of the orthodontic movement, 7 to 10 days after surgery, which is an important concern from an esthetic point of view, The intrusive movements may promote new attachment formation as reported by Melsen etal.,1988 (10).\& Corrente etal 2003 (33).

The orthodontic movement may because stretching of the periodontal ligament fibers creates a natural barrier reducing the down growth of the epithelial cells .Furthermore this stimulation may also increase the turnover of the periodontal ligament cells and improves the possibility of their repopulating the root surface.

In conclusion, this clinical study demonstrated the possibility to perform orthodontic intrusion after proper regenerative periodontal therapy is performed towards intrabony defects of extruded incisors in periodontally involved teeth

Moreover, the realignment of the treated incisors can be achieved by combined ortho-periodontic therapy with radiological bone fill, gain of clinical attachment level, reduction of probing depth and gingival recession. 
Egyptian

Orthodontic Journal

\section{REFERENCES}

1- American Academy of periodontology . Position paper ,Periodontal disease as a potential risk factor for systemic diseases J Periodontol 1998;69:841

2-Emingil LG, Atilla G,Sorsa T,Luoto H, Kirilmaz L,Baylas H. The effect of adjunctive low dose doxycycline therapy on clinical parameters and gingival crevicular fluid matix metalloproteinase -8 levels in chronic periodontitis JPeriodontol 2004;75

3-Demetriou N, Parashis A,Tsami-Pandi A.Relationship between age and clinical symptoms of periodontal disease. Stomatologia (Athenai) 1990;47:231-41

4- Demetriou N, Tsami-Pandi A, Parashis A. Is it possible for periodontal patients to recognize periodontal disease? Stomatologia (Athenai) $1991 ; 47.284-95$

5-Quintero G, Mellonig JT, Cambill VM, Pellen GB.A six month clinical evaluation of decalcified freezed dried bone allografts in periodoal osseous defects. J Periodntol 1982;53:726-30

6-Lekovic V,Kenney EB, Carranza FAJr,Danilovic V .Treatment of class II furcation defect using porous hydroxyapatite in conjunction with polytetrafluroethylene membrane. J Peridontol 1990; 61:575-8

7- Laurell L, Gottlow J, Zubutz M, Persson R. Treatment of intrabony defects by different surgical procedures. A literature review. J Periodontol 1998;69:303-13

8-Naaman NA, Chaptini E, Taha H, Mokbel N. Combined bone grafting and orthodontic treatment of an iatrogenic periodontal defect :A case report with clinical reentry. J Periodontol 2004;75:316-321

9-Re S, Corrente G, Abundo R, Cardaropoli d. Orthodontic treatment in periodontally compromised patients: A 12 year report. Int J Periodontics Restorative Dent 2000;20:31-9

10-Melsen B, Agerbeak N, Erksen J, Terp S. New attachment through periodontal treatment and orthodontic intrusion. Am J Orthod Dentofac Orthop 1988;94:104-6

11- Melsen B, Agerbeak N, Markenstam G. Intrusion of incisors in adult patients with marginal bone loss Am J Orthod Dentofac Orthop 1989;96:232-41 
12- Polson A, Caton J, Polson AP, Nyman S, Novak J, Reed B. Periodontal response after tooth movement into infrabony defects. J Periodontol 1984;55:197-202

13-Gaumet P, Bunvold M, Mc Mahan A. Spontaneous repositioning of pathologically migrated teeth J Periodontol 1999; 70:117-8

14-Nevins M,Wise RJ. The use of orthodontic therapy to alter infrabony pockets. Part II. Int J Periodontics Restorative Dent 1990; 10:199-207

15-Steffensen B, Storey AT. Orthodontic invasive forces in the treatment of periodontally compromised incisors; A case report. Int $\mathrm{J}$ Periodontics Restorative Dent 1993; 13:433-41

16-Thilander B.Infrabony pockets and reduced alveolar bone hight in relation to orthodontic therapy. Semin orthod 1996;2:55-61

17-Re S, Corrente G, Abundo R, Cardaropoli D. Orthodontic movement into bone defects augmented with bovine bone mineral and fibrin sealer: A reentry case report. Int J Periodontics Restorative Dent 2002;22:138-45

18-Re S, Corrente G, Abundo R, Cardaropoli D. The use of intrusive movement to reduce infrabony pocket in adult periodontal patient: A case report. Int $\mathrm{J}$ Periodontics Restorative Dent 2002;22:365-71

19-O'leary TJ, Drake RP, Naylor JE. The plaque control record. J Periodontol $1972 ; 34-8$

20- Ramfojord SP. The periodontal index PDI. J Periodontol 1967;38:602-10

21- Cotellini P, Parto P, Tonetti M.The papilla preservation technique .J Periodonto1995;66:261-66

22-Burstene CJ. Deep overbite correction by intrusion. Am J Orthod $1977 ; 72: 1-22$

23-Braun S, Marcotte MR. Rationale of the segmented approach to orthodontic treatment. Am J Orhod Dentofac Orthop 1995; 108:1-8

24- Ramfojord SP, Morrison Ec, Burrgett CG. Oral hygiene and maintenance of periodontal support. J Periodontol 1982;53:26-3

25- Tonetti MS, Cortllini P. Case selection and treatment consideration of guided tissue regeneration in deep infrabony defects. Curr spin Periodontol 1997; 53:26-30 
26- Laurell L, Goltlow J, Zybutz M, Persson R. Treatment of intrabony defects by different surgical procedures. A literature review. J Periodontol 1998;69:303-13

27-De leonardis D,Garg AK, Pedrazzoli V, Pecora GE. Clinical evaluation of the treatment of class II furcation involvements with bioabsorbable barriers alone or associated with demineralized freez dried bone allografts J Periodontol 1999;70:102-4

28- Wennstrom JL, Lindskog- Stokland B, Nyman S, Thiander B. Periodontal tissue response to orthodontic movement of teeth with infrabony pockets. Am J Orhod Dentofac Orthop 1993; 103:313-9

29- Cardaropli D, Re S, Cortelline G, Abund R. Intrusion of migrated incisors with infrabony defects in adult periodontal patients. Am J Orhod Dentofac Orthop 2001; 120:671-5

30- Aichelmann-Reidy M, Heath C, Reynolds M. Clinical evaluation of calcium sulfate in combination with demineralized freeze-dried bone allograft for treatment of human intraosseous defects. .J Periodontol 2004;75:340-7

31- Geraci TF, Nevins M, Crossetti HW. Reattachment of the periodontium following tooth movement into an osseous defect in a monkey . Int $\mathbf{J}$ Periodontics Restorative Dent 1990;10:184-97

32- Ingber JS. Forced eruption - part I. A method of treating isolated one and two wall infrabony osseous defects- Rationale and case report. JPeriodontol 1974;45:199-206

33- Corrente G, Abundo R, Re S, Cardaropolli D, Cardaropoli G. Orthodontic movement into infrabony defects in patients with advanced periodontal disease : A clinical and radiological study. J Periodontol 2003; 74:1104-9 